\title{
Automatic Conditional Switching (ACS), an Incremental Enhancement to TCP-Reno/RTP to Improve the VoIPv6 Performance
}

\author{
Asaad Abdallah Yousif Malik \\ Abusin \\ Faculty of Computing and \\ Informatics, \\ Multimedia University, \\ Cyberjaya, Selangor, Malaysia
}

\author{
Junaidi Abdullah \\ Faculty of Computing and \\ Informatics, \\ Multimedia University, \\ Cyberjaya, Selangor, Malaysia
}

\author{
Tan Saw Chin \\ Faculty of Computing and \\ Informatics \\ Multimedia University \\ Cyberjaya, Selangor, Malaysia
}

\begin{abstract}
In this research work an Automatic Conditional Switching Protocol (ACSP) is proposed, which is a conditional switching method between Delay based TCP-Reno and RTP (Real-Time Transport Protocol). It is a delay constrained method based on the VoIPv6 delay limit stated by the Internet Engineering Task Forth (IETF), which is 150 milliseconds in accordance with the ITU G.114 standard recommendation (G.114 International Telecommunication Union, 1994) which recommended that the permissible amount of delay in VoIP to be less than or equal to $\mathbf{1 5 0}$ milliseconds in order to maintain the voice quality. This proposed Automatic Control Switching (ACS) should be implemented in the routers and switches in the protocol layer of the VoIPv6 network to improve VoIPv6 performance. The system can be defined simply as combination of TCP Reno/UDP (RTP) with Automatic Conditional Switching. This Automatic Conditional Switching is based on Delay based Congestion Avoidance (DCA).
\end{abstract}

Keywords-TCP Reno; VoIPv6 Performance; VoIPv6 improvements

\section{INTRODUCTION}

The Automatic Conditional Switching Protocol (ACSP) is a Delay Avoidance, Two Pass method for VoIPv6 which should be implemented in the routers and switches in the protocol layer of the VoIPv6 network in order to improve VoIPv6 performance utilizing DCA (Delay based Congestion Avoidance) as a driver. A report on the performance of the two protocols utilized in ACS is presented in [26], and a proposal to carry TCP traffic over UDP was proposed in [27], which was supported by review on the different TCP variants, and how they can support the voice traffic, these TCP variants were discussed in [29]. [30] compared between the different TCP flavors among which ACS utilized TCP Reno to work conditionally with RTP in order to produce the resultant ACS packets.

\section{ReSEARCH Methodology, ANALysis, AND DESIGN}

A. Key Concepts in Delay-based TCP as Part of The

Switching Process in this Proposed ACS

The collection of the TCP packets congestion control techniques that are correlated with TCP/Reno are considered as a performance improvement measures which are mainly (slow- start and congestion avoidance) which can provide network saturation based on improved characteristics. The enhancement method to improve VoIPv6 performance ACS is proposing to use this delay based TCP in one hand and RTP (UDP) on the other hand and applies a conditional real time switching between both based on conditional switching criteria. In order to establish this, there is a need to apply congestion controls in VoIPv6 traffic, these congestion conditions according to [2] are:

- Delay based TCP

- Loss based TCP

- TCP congestion control

- Mixed loss/delay passed congestion control

- Explicit congestion notification

- Prediction of the lost VoIPv6 packets.

Each of the above six congestion control conditions is subjective to enhancement, and can be proposed as a research basement for further enhancements in VoIPv6 performance. This research work made enhancements on the proposal made by [2], and implemented new VoIPv6 improvement technique that is Delay based TCP/RTP (UDP) with conditional switching capability to be implemented as an ACS in the IPv6 network routers and switches. As a step to support this proposal, it is important also to study, test, and evaluate the overhead imposed by this improvement suggestion and then look for ways to reduce it. [28] discussed the challenges in network chatting and Sending Data including voice. A very important statement from [2] is that, in order to attain high level of deployment of new TCP enhancements such as this research work proposed ACS, there must exist sufficient encouraging economic factors in order to adopt such proposed measures, in some cases these encouraging economic factors to deploy new improvements are weak or may result in temporary sacrifice of the available resources for users prepay for a considerable length of time before its deployment become practically popular in the network. The VoIPv6 performance improvement proposal in this research work is of high economic value since the economic value of long distance 
calls, business transactions, and electronic transactions in general, besides the real-time transactions and the social media is high. Both the research work carried out by [3], and the proposed ACS in this research work utilizes the benefit of Delay based Congestion Avoidance (DCA) added by studying the performance of Primal Dual Schemes for Congestion Controls in busy networks with dynamic stream such as VoIPv6 network.

In order to test ACS in this research work, a VoIPv6 Client/Server transactions were monitored and the inter-arrival time of the voice packets were captured using FreeBSD which is similar to [4] who studied Client/Server transactions for sensitive traffics such as VoIPv6, in which the scaling of virtual worlds in the age of cloud computing is complicated by the problem of efficiently directing client-server traffic in the service of dynamic compute resources in a different approach from this research work emphasizing on cloud computing proposing a model in which software defined networking and compact encoding of important data in packet headers were combined to make a fast, scalable, high capacity proxy server that can hide the server infrastructure while fitting well with the structure and design of modern network service providers.

A DCA (Delay based Congestion Avoidance) algorithm cannot reliably assess the congestion level at the router; this is due to the Short-term queue fluctuations. The measured data in [2] was collected in the year 1999 and the results were published in the year 2003 provided that the observed dynamics from their conducted tests were representing June 2003 public Internet quality and behavior. This also is pointing clearly to the fact that improvement in VoIPv6 performance is of cumulative nature, and this research work studied these accumulated factors such as VoIPv6 packet delay, and the performance of the network related functions, based on Client/Server tests conducted in the years 2013 and 2014. In order to implement ACS it is important to study the interrelation between packet delay and TCP Round Trip Time for the data packets, this is mainly because the TCP constrained RTT congestion verifier exactly track the level of packets congestion related to packet delay over busy network with high packets stream forwarding including VoIPv6 stream. A DCA (Delay based Congestion Avoidance) algorithm cannot efficiently assess the level of congestion at the router; this is due to the Short-term queue fluctuations, and the performance improvement in any of the network levels will contribute to the overall VoIPv6 network performance improvement; in this point this research work focus on packet delay and packet delay avoidance.

[5] coined the term Delay-based Congestion Avoidance (DCA), he admitted that his DCA algorithm is not sufficient for practical networks, but his work provided the foundation and framework for future research on DCA and interrelated mechanisms including ACS as an enhancement in the protocol level of the VoIPv6 network, introducing TCP/Vegas compared to this research work which utilized TCP/Reno as part of ACS. Their studies were based on Internet paths that existed in the early 1990s which generally involved at least one-To-one speed link, and allowing any given flow to consume a significant fraction of available bandwidth. The network performance improvement methodology proposed by [5] discussed congestion avoidance algorithms, this congestion avoidance is the scientific basement of Delay based TCP, and Delay based TCP is the basement for this research work main contribution (ACS Protocol), supporting the VoIP continuous evaluation, and improvement effort as in [22].

According to [2], a substitutional packets congestion control mechanism ought to be a TCP-compatible resulting in a similar performance to that achieved by a likewise network host across resembling network path to TCP characteristics. As this research work is an incremental enhancement to TCP, and accordingly any proposed incremental performance improvement in TCP that coincide with the following requirements will be competently deployable, and accordingly this research works proposed ACS deployment in the network can be evaluated and assessed according to the following criteria:

- The incremental enhancement ought to increase the number of transmitted TCP packets that adopts the performance enhancement.

- The incremental enhancement ought not to negatively affect the performance of other TCP packets streams competing in the utilization of the same network route through which the enhanced TCP flow is being transmitted, that is in order to attain better utilization of the network available bandwidth without negatively affecting other TCP flows.

- The incremental enhancement need to change the TCP client, and the above two properties must be attained without considering the number of improvements in the TCP connections through the same Client/Server path, consequently these properties must be maintained even if there exists just a single enhanced flow in the client/Server connection. In the wide spectrum of the global Internet for the purpose of supporting global implementation of TCP incremental enhancements, a substantial enough encouraging reasons must be present leading to adapt them, in the case when these encouraging factors are less significant, or causing short term cut of resources for users before its complete deployment, then the resultant final wide deployment of such protocols will be negatively influenced.

[2] described the main features of the Delay based Congestion Avoidance (DCA):

- The DCA strengthen the performance of the TCP/Reno protocol.

- DCA monitors, and trace the TCP packet Round Trip Times (RTTs) allowing the algorithm to reside holistically within the TCP server.

- The Round Tripe Time continuous changes are looked into as a consequence of changes in queuing delays occurred to the timed out packets.

- Based on Round Trip Time changes, the Delay based Congestion Avoidance (DCA) makes the necessary 
congestion decisions that reduce the packets transmission rate by adjusting the TCP congestion window.

[5] invented the term delay-based congestion avoidance, he confirmed that his proposed DCA was not substantially applicable for a practical packetized network; but DCA provided the platform, and benchmark for the on-progress research work on Delay based Congestion Avoidance (DCA) towards making it practically applicable. This research work proposed ACS protocol as an improvement in Delay Tolerate Networks (DTN) provided that ACS is governed by the 150 milliseconds delay limit or hit value, as this research try to utilize (R. Jain, 1989) idea in order to produce the Conditional Switching Protocol (CSP).

[25] encouraged improvements during the World IPv6 Day by AMPST of Australia in the different level of the network to support IPv6 applications. Research work in [2] was utilized as part of this research work proposed ACS as it assessed the possibility in TCP controlled congestion sampling algorithm in RTT. Forwarded by utilized it in predicting the possibly coming packet loss occurrence which can be part of performance improvements in packet based real time networks, and for this reason, this research work used RTT-based congestion sampling algorithm as part of DCA in the proposed ACS. Source to destination packet delay and loss attitude over the Internet, as these factors are considered the main two considerations in VoIPv6 performance improvements. A finding result in [2] showed that the occurrence of packet loss was found to be interrelated over packets inter-arrival time values up to 200 milliseconds with loss correlation up to one second ignoring the interrelationship between the rise in RTT and packet loss ignoring the existing interrelationship involving loss conditioned delay that occurs immediately after a packet loss. The motivations of [2] were similar to this research work in the sense that they studied the protocol level locking for possible improvements. When examining the occurrence of packet loss, the result will be that the RTT that tend to increase the surrounding packet loss will live for short time. Loss processes are optimistically interpreted as chunks of data during which there exists a very short-term stops, instead of short chunks of data over which congested router's buffer is congested with packets that could be discarded.

The research outcome from [2] is that DCA working alone is not substantially enough to improve TCP performance and is accordingly conditionally not applicable to be deployed as an increment to TCP unless DCA is supported by other techniques such as this research work proposed ACS since this research work tries to improve DCA by adding it accumulatively in the switching between TCP Reno and RTP, to work together in a joint performance improvement effort. Besides the TCP congestion control aspects, the research led by the internet research bodies of concern including the IETF, and the IF has also been extensively verifying, examining, and applying TCP algorithms for real-time packet traffic applications including voice, this is exactly what this research work try to achieve by proposing ACSP which can be considered a TCP/RTPFriendly algorithm to improve VoIPv6 performance. The performance of the deployable TCP-friendly algorithms can be similar to TCP when they utilize Additive Increase
Multiplicative Decrease (AIMD) behavior with the same features as TCP. The TCP potentially deployable methods, such as this research work proposed ACSP, are subjective to further assessment referring to steady-state or dynamic behavior, an illustrative example here is the TCP-friendly rate control (TFRC) technique which responds to a single packet loss by reducing the packets transmission rate to values smaller than TCP packets transmission rate. The TCP deployable incremental algorithms are targeting to be TCP compatible by emulating the TCP behavior, with the TCP main features such as slow start, congestion avoidance, and exponential timeout back off in addition to self-clocking. All rate-based protocols that fix their rates based on a computation, including this research work proposed ACS incremental enhancement protocols, ought to include a measured RTT in order mainly to add an element of delay-based congestion avoidance (DCA) to their algorithms.

[2] proposed techniques based on hypotheses on implicit packets congestion feedback based on delay instead of packet loss or additional to packet loss, and this can positively improve the performance of the global IP network in case high number of IP traffic adopted the same algorithm emphasizing mainly on packet delay rather than packet loss. The expected outcome would be the same as what inspired the alternative best effort (ABE) proposal, which is a network that provides packets forwarding service that dedicated to attain less amount of delay and less throughput; such service expected to be beneficial for multimedia applications that are very sensitive to delay such as VoIPv6. This encourages studying shortest bath selection for VoIP packets and the hop beer hop behavior in DCA as this DCA is the basement for this research work proposed Automatic Conditional Switching (ACS).

In their experiments [2] used TCP-Dump to trace the TCP connection in a similar manner as in this research work experiments in which Client/Server connections were established, traced, and monitored from the time the connection was established between the VoIPv6 client and the VoIPv6 server to sending VoIPv6 packets stream, the TCP sender experiments as well as the trace point was a $333-\mathrm{MHz}$ PC running FreeBSD. FreeBSD was the same performance testing medium used in this research work. The machine used in [2] consists of 3COM PCI Ethernet adapter and was attached to North Carolina state university (USA) campus network through a $10-\mathrm{Mb} / \mathrm{s}$ Ethernet connection. These experiments were similar to this research work experiments conducted at Multimedia University (MMU Malaysia) intranet in the year 2013 in which a developed Client/Server codes were used to capture the arrival and the inter-arrival time of a real time VoIPv6 packet samples, in addition to that the experiments carried out in this research work similarly used FreeBSD as a testing tool. The packets queue management techniques that can be injected in the routers can contribute in rendering enhanced performance, and this research work main contribution ACS is proposed to be applied in the routers and switches of the VoIPv6 network.

[6] presented a simulation scenario describing the performance assessment of the two main transport layer protocols TCP and UDP using two queue management methods; Random Early Detection (RED) and Drop Tail, 
mainly to trace the network throughput, queuing delay, packet drop rate and bandwidth utilization studying the effect of packets buffering on each performance measurement by simulating TCP, UDP and shared topology network scenarios by considering different number of client topologies and covering also the effect of performance in TCP and UDP with increasing the number of clients, such simulation work can be beneficial for implementing the packets queue control mechanisms in the router based on the traffic type and the available network bandwidth, an interrelated terms to packets management in the network are TCP, UDP, drop tail, RED queue, bandwidth delay product, throughput, and end-to-end delay, such effects can be considered as the major factors affecting VoIPv6 performance.

\section{B. Existing Measures Contributing to VoIPv6 Performance Improvement}

The way is open for new improvements in IPv6 and the accompanying applications, this is according to [24], ACS utilized measures contributing directly to the VoIPv6 performance improvements, which are mainly measured to remedy the collegian, contention, congestion, and queuing of voice packets. Performance measurements are needed to measure the collegian, contention, congestion, and queuing of the voice packets in terms of percentage of the network loss and collegian rate. One technique is needed to reduce or avoid collegian, and in case the technique is good for normal traffic then it can be tested in highly congested traffic in order to develop measures to reduce collegian, and avoid delay. [23] supported research, and discoveries for VoIP supported by ACS. The proposed ACS utilizes User Datagram Protocol (UDP) in its enhanced RTP form, and Delay based TCP which is using DCA jointly as an enhancement for the two commonly used protocols on the Internet (TCP and UDP) for them to work concurrently and conditionally. This is an improvement contribution in this research work because UDP, and its refined form (RTP) used not to send important data such as web pages, and database information, and it is commonly used for packetized voice and video Streaming, such as Windows Media audio files (WMA), Real Player (RP). The constrained packet transmission was used in this research work proposed ACS conditionally, and when the condition satisfied the switching to TCP-Reno will take place, and with the continuous real-time monitoring of the voice speed (less than or equal to 150 Milliseconds) switching takes place back and forth conditionally.

Many VoIPv6 current applications uses UDP because it offers speed, and faster processing for the real time traffic. The reason UDP is faster than TCP is because there is no any form of flow control or error correction. The data sent over the Internet is affected by collisions according to witch errors in packets forwarding will be present. UDP is only concerned with packets speed, sacrificing the quality, and this is the main reason behind the lake of quality in streaming media, which is a hot research aria as this research work concern. In this research work proposed ACS, in the switching process, a consideration for future research work can be to figure and correlate by obtaining a correlation function, between the overall total delay and packet delay, and loss relationship with the routers buffer size and the per hop behavior in VoIPv6

Identify applicable sponsor/s here. If no sponsors, delete this text box (sponsors). traffic, keeping in mind that ACS avoids packet loss focusing on improving the VoIPv6 performance utilizing packet delay in the form of ACS as this research work main finding.

The Real Time Transport Protocol (RTP, RTCP) is utilized as part of the switching in this research work proposed ACS as illustrated in Fig. 1. In [7], they specified set of extensions to the base Real-Time Transport protocol which was specified in RFC 3550, according to which RTP is a collection of network transport functions that are applicable for applications transmitting real-time data such as VoIPv6 stream transmitted between network client and a server. When comparing this research work proposed ACS with the proposed TCP/UDP router in [8], it is noted that their performance improvement suggestion is based on developing a linear time-delay system model which can be derived by obtaining packets flow stability conditions with elementary router parameters using the 2-D Laplace-Z transform technique leading to deriving parameter setting for TCP/UDP routers in order to improve the network congestion control based on 2-D local stability conditions, and this can be achieved through applying serial of simulations which verified that their proposed router can attain the desirable packets congestion control by utilizing the stability conditions, proving that performance comparison for different TCP/UDP network scenarios with the traffic stability conditions can contribute to performance improvements as in this research work the reference stability condition is the 150 milliseconds overall cumulative delay limit hit value. Up to the year 2014, according to [9], most multimedia applications, including VoIPv6 services, and applications utilizes a combination of Real-Time Transport Protocol (RTP) and User Datagram Protocol (UDP). ACS in this research work is a switching mechanism applied in a combination of TCP Reno and RTP. The developed application programs at the source end format payload data into RTP packets using RTP specifications and dispatch them using unreliable UDP along a single path, multi-path transport, this means that up to 2014 there is no suggestion to switch between TCP and UDP which is considered an important on the efficiency of voice data delivery.

\section{The Proposed ACS Compared to New Trends in VoIPv6 Performance Improvement Methods}

A step towards VoIPv6 performance improvement is through continuous performance testing trails, and for the improvement in the protocol layer of the VoIPv6 network, one of the improvement methods (as they are many ways in the three VoIPv6 network layers), is the removal of the checksum operation at the network layer which is not enough to recover the long address processing time. The IPv6 header is considered simpler when compared with IPv4, and the simpler IPv6 header does not help in this case because experiments in [10] were conducted within MMU (Multimedia University of Malaysia) intranet in local loop intranet environment. From the theoretical point of view, it will enhance the end-to-end performance if there are many intermediate hosts since there will be less processing in the intermediate hosts and nods due to the distribution of tasks. IPv6 uses smaller send and receive buffer compared to IPv4, this affect the conducted performance tests significantly since small buffer become full faster. IPv6 is still considered improving and in its evolving stage, this is 
according to the IETF. And ITU G.711, and G.744 of the ITU which expect IPv6 to reach its maturity by the year 2050 welcoming continuous requests for comments to be published as RFCs. The IPv6 KAME stack is under continuous development and frequently being changed and updated confirming the fact that the current release of IPv6 is not the best all the time, and a research work can be carried out in investigating the performance of the implementation of the IPv6 stack. KAME IPv6 stack implementation needed continually to be improved to the most optimal level to give better performance in the associated applications. These tests can be repeated in different network conditions and highlight the differences and similarities. The IPv6 features supporting real-time traffic including VoIPv6 stream can be clearly noticed in the current internet, which are needed due to the evolving enhancements in IPv6 network with the introduction of Broadband internet service which can contribute to performance improvements in the real-time applications under IPv6.

\section{DESCRIPTION OF THE PROPOSED ACS MODEL}

This research work proposed ACS is to be implemented in the routers and switches of the VoIPv6 network. An illustrative diagram for ACS is shown in Fig. 1. ACS treats VoIPv6 packets under TCP Reno then apply Congestion/Delay based TCP as in [11] from which the resultant packets to be injected to TCP-Dump to trace the TCP connection and then use RTTbased congestion sampling as in [2]. Then use Router scheme with classifier and access control to separate UDP from TCP traffic using linear time delay system model based on stability condition which is the VoIPv6 maximum delay limit which is 150 milliseconds. Then use Delay based (for voice packets)/Loss based TCP congestion Avoidance (DCA) and loss based TCP/UDP router (for the associated data packets). The last step is the switching step, which is to use Linear Time delay system to continuously compare the total delay with 150 Milliseconds, this is the moment of the conditional switching when the total delay is less than or equal 150 milliseconds then to use TCP Reno, else if the total delay is greater than 150 milliseconds then to use the Real-time Transport Protocol (RTP) in order to produce the resultant processed ACS packet.

There are two options to conduct ACS tests in real time:

1) Software Virtual Machine in ready available commercial software

2) Hardware development, which can be done by following the steps in the ACS descriptive diagram as in Fig. 1

In this research work, the ACS switching was tested based on FreeBSD captured VoIPv6 data (Packets Inter-arrivals) as shown in Fig. 2. ACS switching was tested using Mat Lab. In order to accomplish this goal this research followed the following steps while using Mat Lab:

1) Read data which was put in Excel sheets.

2) Count the number of rows and columns

3) Then if conditional statement, in case the incoming tested value overall delay is less than or equal to 150 milliseconds then use TCP Reno, else if the overall delay is greater than 150 milliseconds then switch to RTP and be in continuous monitoring alert mode to take the ACS decision. In case the intention is to make the tests in real time then the steps as in the diagram in Fig. 1 should be followed. For performing the ACS testing in real time then the following equipment's are needed:

- Analog to digital converter to produce the digitized voice in real time.

- Traffic shaper to shape the packets in IPv6

- Perform ACS.

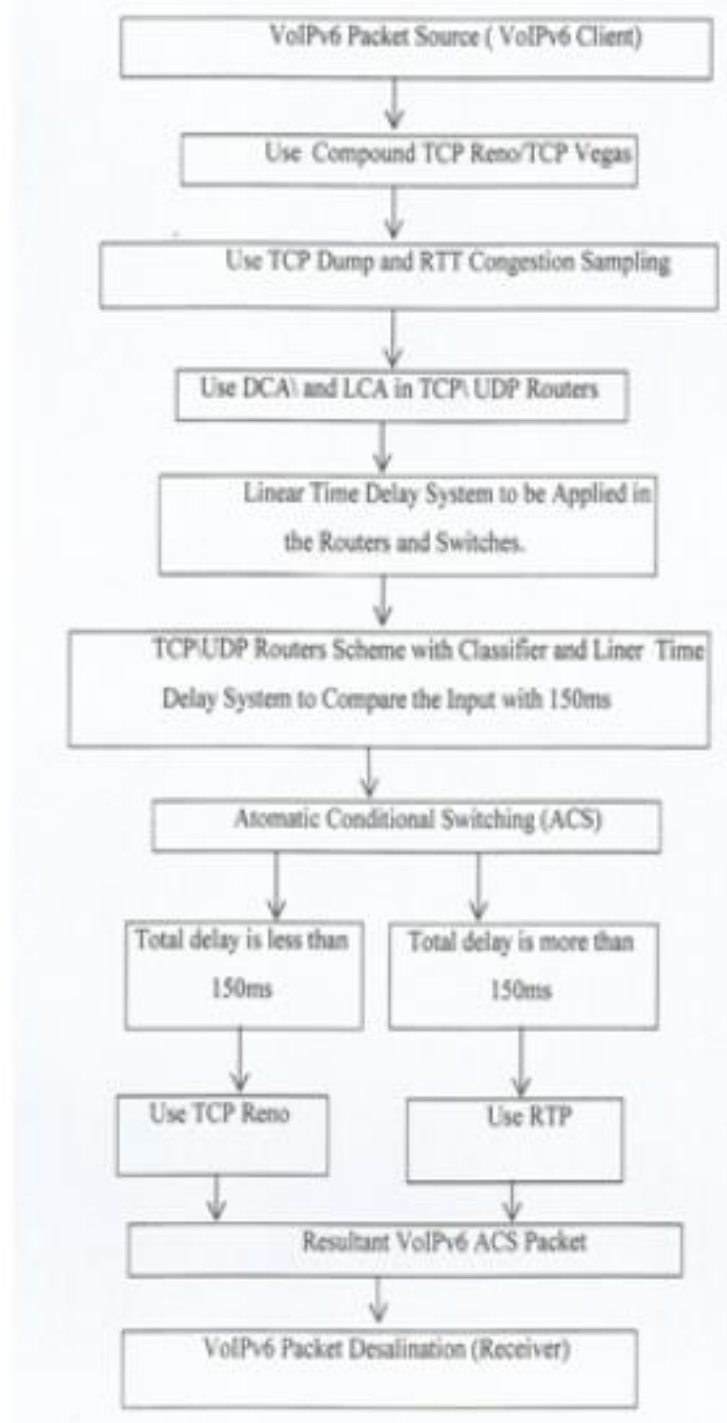

Fig. 1. ACS Descriptive Model Diagram, Mathematical Model Using Laplace Z Transform is used to Represent the Switching in ACS

\section{A. ACS Validation}

ACS validation and testing is of concern here as it can be a platform for further improvements towards ACS practical implementation, in this context a future work can be the usage of Laplace Transform to represent the switching mathematically. 


\section{B. Mat Lab Code Describing ACS on a Captured FreeBSD VoIPv6 Data}

The following Mat Lab code was developed, and used to perform the switching in ACS on captured VoIPv6 inter-arrival time data values captured using FreeBSD. The following Mat Lab code reads the VoIPv6 inter-arrivals data values in milliseconds stored in Excel files:

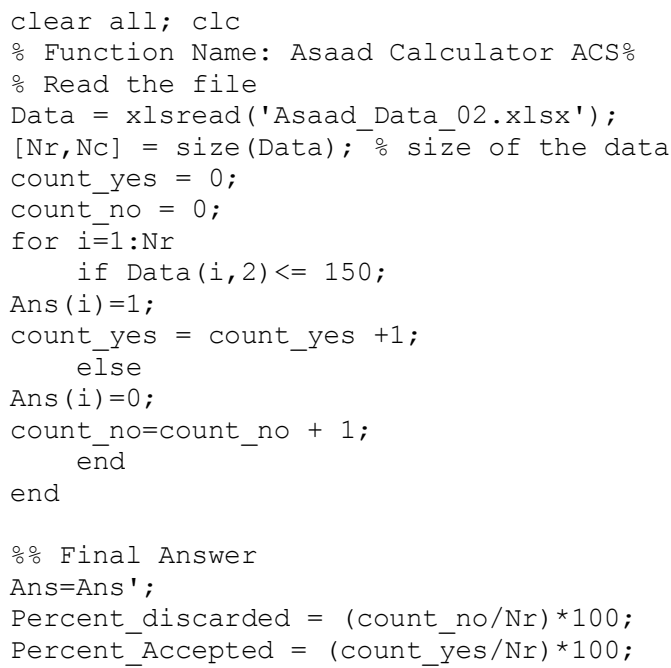

C. Test Results for ACS Validation Using Mat Lab Applied to the VoIPv6 Packets Inter-arrival Data

In Table 1 the number of yes indicates that the Inter-Arrival time for the voice packets is less than or equal to 150 milliseconds. The number of No indicates that the Inter-Arrival time for the VoIPv6 packets is greater than the 150 milliseconds hit value.

TABLE. I. RESUlt ANALYSIS USING MAT LAB For CAPTURED VoIPv6 PACKETS DATA

\begin{tabular}{|l|l|l|l|l|}
\hline \multirow{2}{*}{$\begin{array}{l}\text { Data } \\
\text { Name }\end{array}$} & \multicolumn{4}{|l|}{ Table Column Head } \\
\cline { 2 - 5 } & Number of Yes & Number of No & $\begin{array}{l}\text { Percentage } \\
\text { Accepted }\end{array}$ & $\begin{array}{l}\text { Percentage } \\
\text { Discarded }\end{array}$ \\
\hline Test 1 & 677 & 323 & $67.7 \%$ & $32.3 \%$ \\
\hline Test 2 & 846 & 154 & $84.6 \%$ & $15.4 \%$ \\
\hline Test 3 & 996 & 4 & $99.6 \%$ & $0.4 \%$ \\
\hline
\end{tabular}

\section{Testing the Switching Possibility in ACS Based on DNS}

The performance of the IPv6 addressing system to surf the internet is based on the communication between source and destination IP addresses which is based on spotting the IPv6 address and the port number, besides the Domain Name System (DNS) that is involved in the background applications accompanying the VoIPv6 stream of packets in which the client is continuously monitoring and recording the arrival and inter-Arrival time of the IPv6 packets, and ACS is based on continuous monitoring for the 150 milliseconds voice packets hit value in order to decide performing the switching back and forth conditionally between TCP Reno and RTP. The recorded background information that is required as condition to perform the switching, is lengthy for UDP in order to take the decision to perform ACS, and the DNS steps can be:
1) The server responds with specifying opcode to have client switch to TCP Reno.

2) When the server is not responding, the client re-transmit over TCP.

3) The server respond by opening TCP connection to client Network Address Translation (NAT).

4) The client now that the given query should be run prior over TCP, for this reason RTP cannot be used in the first place.

5) DNS tiny chunks turn UDP into TCP conditionally utilizing DNS and Name server searching trails.

The conditional switching based on DNS is known in the internet used to block the surfing of undesirable web sites, but the automatic conditional switching between protocols is not practically known yet which is proposed as ACS in this research work which needs more verification before its practical deployment. ACS also is not deployed in the current versions of Opnet (current version 17.5) and Netsim (version 10). These WAN and network simulators are mainly dedicated to perform the simulation only for delay, packet loss, and jitter targeting mainly these network impairments, and needed to be upgraded to newer versions that can allow for Automatic Conditional Switching between TCP Reno and RTP.

\section{E. How to do the switching Between TCP Reno and RTP?}

According to this research work it can be achieved by encapsulating TCP Reno on RTP, and the encapsulation, can be achieved using Network Address Translation NAT defined in RFC-1631. Originally NAT was designed not to permit the depletion of IPv4 address, it is proposed here to be used in encapsulating TCP Reno into RTP in order to facilitate the switching between them. NAT is a method to remap one IP address space into another by modifying network address information in the Internet Protocol (IP) datagram packet headers during their transit across a network traffic router. The port forwarding is needed in NAT, in addition to the category network address including the Address Space. Some researchers criticize NAT, and think to avoid it in the IP network design and implementation because it breaks the connectivity between hosts especially in packetized VoIPv6 networks. [13] defined NAT when a packet is received from an internal host, the NAT process searches for the matching source address-source port combination in the port-mapping table which contains a list of inner local port numbers that get translated into particular mapped ports, and in case the entry is not found for the received source port, a new port translation entry is created and the new port is allocated for the received source port. The source IP address is replaced with a translated global address from the translation table or NAT address pool and the source port is replaced with port number from the portmapping table. At the end of the NAT process, the voice packet is sent out with the translated source IP address and source port number, and the time needed for this process is named as practical delay in NAT Router.

\section{F. Practical Delay in NAT Router and its impact on ACS}

The delay in NAT router needed to be reduced to the minimum value with a minimum possible contribution to the 150 milliseconds less overall delay in VoIPv6 as recommended by the G.711 of the ITU. The practical network delay with NAT network according to [14], when ping a server which 
usually requires 20 to 30 milliseconds for NAT, the Virtual Box will get 20 to 30 milliseconds for the first three pings (acceptable for voice), then 500 milliseconds (unacceptable for voice) for the next 3 pings which is overcome by ACS, then 500 milliseconds for the next 3 pins (unacceptable for voice), and then back to 20-30 milliseconds (acceptable for voice). Because of NAT nature the first switching by ACS after receiving three consecutive NAT packets should be freezed for a predetermined length of time before the next switching. RTP as part of ACS runs over UDP, for this reason a VoIPv6 packet has a 20 bytes IP address distributed as 8 bytes UDP packets, and RTP 12 bytes packets headers added to the encapsulated voice payload. The Digital Signal Processors (DSP) creates a package from each10 milliseconds of Analog voice, and two of those packages are transported within one IP packet. A 20 milliseconds resultant packets of voice traffic is common in one IP packet. The number of bytes resulting from 20 milliseconds from Analog voice depend on the codec slandered used. The G.711 which produces $64 \mathrm{Kbps}$, produces 160 bytes from 20 milliseconds of Analog voice, and G.729, which generates $8 \mathrm{Kbps}$, out of which is 20 bytes for 20 milliseconds of Analog voice signal. The RTP, UDP, and IP headers, with total of 40 bytes, are added to the voice bytes (160 bytes for G.711 and 20 bytes for G.729) before the whole group is encapsulated in the Layer 2 frame and transmitted.

In case the Network Layer 2 overhead is not considered, considering only the overhead resulted by RTP, UDP, and IP, it can be notable that the needed bandwidth is more than the bandwidth that is needed for the voice payload, as when using the G.711 codec, the required bandwidth for voice is 64 Kilobytes per Seconds, but with 25 percent added overhead of IP, UDP, and RTP, the required bandwidth increases to 80 Kbps. In case the G.729 is used, the bandwidth required for voice packets is $8 \mathrm{Kbps}$, but with the added 200 percent overhead imposed by IP, UDP, and RTP, the required bandwidth increases to $24 \mathrm{Kbps}$. The overhead imposed by the Layer 2 protocol provided that any other techniques such as tunnelling or adding security mechanisms is not included.

In order to reduce the header overhead imposed by IP, UDP, and RTP the Compressed RTP (cRTP) can be used, which is also called RTP header compression, as cRTP reduces the overhead imposed by all IP, UDP, and RTP protocol headers. cRTP should be applied on the sending and receiving ends of the voice packets, and both agree to a hash number that is associated with the 40 bytes of IP, UDP, and TCP headers. In cRTP most of the fields in the IP, UDP, and RTP headers do not change among the packets of a common flow. After the initial NAT packet with all the headers is submitted, some packets that do not carry the 40 bytes of headers, and instead, the packets carry the hash number that is associated with those 40 bytes with their sequence number built in the hash. The main difference among the headers of a packet flow is the header checksum which is UDP checksum, and in case cRTP does not use this checksum, the size of the overhead is reduced from 40 bytes to 2 bytes. In case the checksum is used, the 40 bytes overhead is reduced to 4 bytes, and in case during transmission of packets, a cRTP sender realized that a packet header has changed from the normal form, then all the header instead of the hash is submitted. The benefit of using cRTP with smaller payloads such as digitized voice is more recognized than other large payloads.

Fig. 2 describes the steps in the switching by encapsulating TCP Reno over RTP in order to improve VoIPv6 performance. The starting of the process is the voice packets source exchanging voice packets arrival and inter arrival information within the network mainly with the server. An application program is needed to shape the voice packets in IPv6 followed by using TCP Reno as in RFC 793. A real time bottleneck is applied in order to monitor the arrival and inter arrival time of the voice packets in order to perform the switching conditionally.

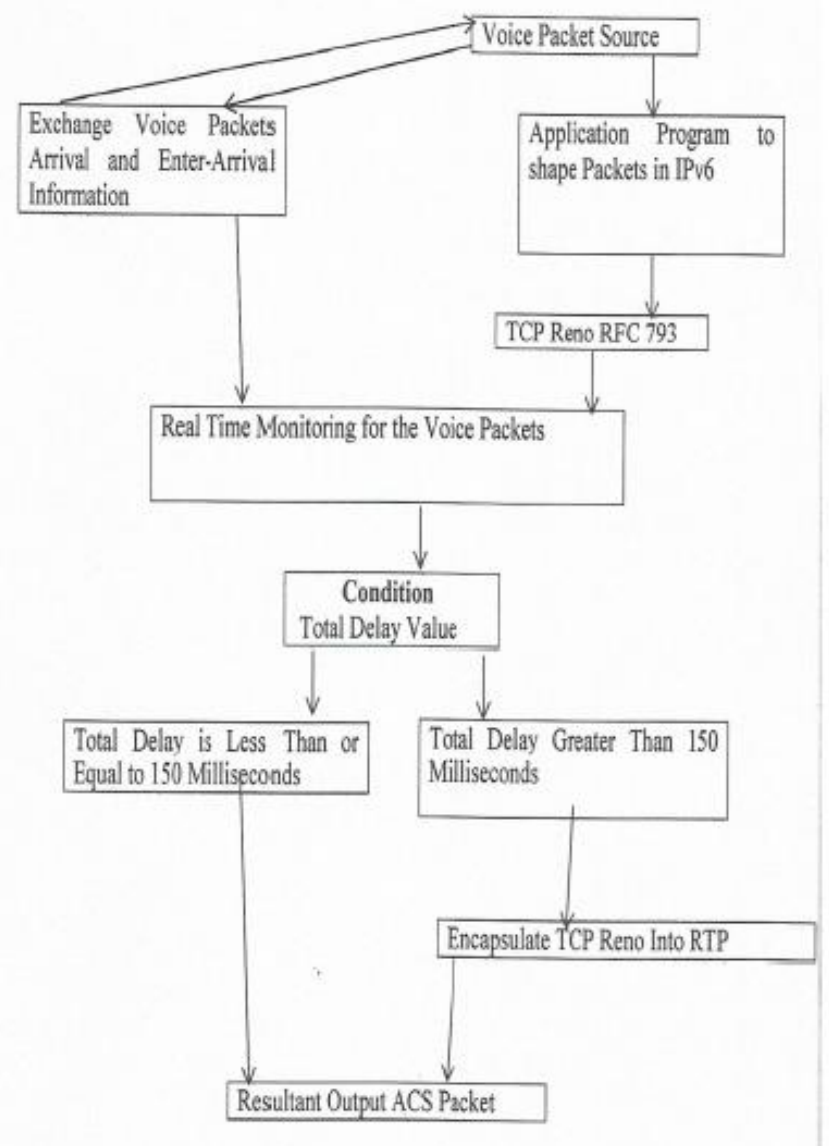

Fig. 2. The steps in the switching by encapsulating TCP Reno over RTP in order to improve VoIPv6 performance

\section{THIS RESEARCH WORK MAIN VOIPV6 IMPROVEMENT METHOD (ACS)}

This research work's main VoIPv6 improvement idea is a smart conditional switching capability for the VoIPv6 traffic as an incremental enhancement to a combination of TCP Reno and RTP, which can be described precisely as, Delay-based TCP-UDP Selective Dual Stack (TCP Reno/UDP RTP) routers scheme with classifying, selecting and switching capability. This ACS model idea resemble the automatic conditional switching in the automatic gearing system in the automatic car, whereby the continuous monitoring in the automatic gear is for the car speed and in this research work proposed ACS it is for the voice speed in milliseconds. Likewise the existence of dual 
stack IPv4/IPv6 idea, this research work proposes the dual existence of Delay-based TCP Reno-RTP Selective Conditional Switching Dual Stack routers to improve VoIPv6 performance.

According to [9], in the year 2014, most multimedia applications utilize a combination of real-time transport protocol (RTP) and user datagram protocol (UDP), this statement support the uniqueness and the genuineness of this research work proposed ACS as a smart back and force conditional switching between RTP and TCP Reno. When comparing this research work proposed ACS with the proposed TCP/UDP router in [8], it can be noted that there proposal is a packets congestion avoidance scheme that can be expressed by a linear time-delay system model after deriving some stability conditions with simple router parameters by utilizing the 2-D Laplace- $Z$ transform technique.

This research work proposed ACS is based on the following:

- A criteria in the VoIPv6 network that contributes to good or bad voice quality, and accordingly finding new switching criteria based on the 150 milliseconds less possible voice delay limit.

- The correct switching decision in the VoIPv6 network with reference to ACS can be able to improve the performance in VoIPv6 utilizing Automatic Conditional Switching Protocol or ACS which is this research work main contribution.

- Formulate a model for the switching process, which is a design to switch back and forth automatically between RTP and TCP Reno.

ACS can be considered as a new contribution when compared with the statement from [9] which stated that, in the year 2014, most multimedia applications utilize a combination of real-time transport protocol (RTP) and User Datagram Protocol (UDP).

\section{A. The Scientific Basement for ACS}

Based on [8] proposal, this research work propose ACS, their proposed TCP/UDP router can be described as linear time-delay system model which augmenting stability conditions in the network with elementary router parameters by utilizing the 2-D Laplace- $Z$ transform mechanism, by proposing parameter setting for TCP/UDP routers in order to improve the network congestion control based on 2-D local stability conditions. A serial of simulation results obtained by [8] verified that their proposed router can attain good level of network congestion control concerning the stability conditions considering performance comparison for different TCP/UDP network scenarios with the associated stability conditions. In [2], they were looking for incremental improvements to TCP to support real-time applications, such incremental improvements as TCP Reno and TCP/Vegas. TCP Vegas attempt to utilize the congestion information contained in packet round-trip time (RTT) samples. TCP Reno instead of TCP Vegas was used as part of this research work proposed ACS due to the sensitivity of VoIPv6 stream to packet loss.
The Real Time transport Protocol (RTP) specified in (RFC3550) was used as part of this research work proposed ACS in the switching process. This RTP was specified by (Microsoft Extensions, Thursday, May 15, 2014) which described the Real-Time Transport Protocol (RTP/RTCP) according to which RTPME is a set of extensions, as similar as this research work proposed ACS which is also considered as an extension or increment to TCP Reno/UDP in addition to the base Real-Time Transport Protocol (RTP) specified by the IETF in (RFC3550). RTP is a set of network transport functions suitable for applications transmitting real-time data, such as audio and video, across multimedia endpoints including VoIPv6.

[2] focused on delay-based congestion avoidance algorithms (DCA), like TCP/Vegas, which attempt to utilize the congestion information contained in packet round-trip time (RTT) samples in an attempt to look for performance improvement possibilities through measurement and simulation showing evidence suggesting that a single deployment of DCA, which is a TCP connection enhanced with a DCA algorithm, is not a viable enhancement to TCP over High-speed paths, this research work tried to overcome this by utilizing combination of Refined TCP, and RTP, and applying ACS between them.

[11] highlighted that some efforts combined the features of loss-based and delay-based algorithms, in this context this research work proposed (ACS) can be considered utilizing DCA to achieve fair bandwidth allocation and fairness among flows, and consequently improve VoIPv6 performance. A comparative analysis between different flavors of TCP congestion control namely Standard TCP congestion control (TCP Reno) which is the TCP flavor used in this research work proposed ACS, loss-based TCP congestion control (Highspeed TCP, Scalable TCP, CUBIC TCP), delay-based TCP congestion control (TCP Vegas) and mixed loss-delay based TCP congestion control (Compound TCP). These TCP flavors were presented In terms of congestion window versus elapsed time after the connection is established. Work in [11] is the foundation for this research work proposed improvement method to enhance VoIPv6 performance (ACS), which can be considered as Delay Based TCP/UDP Congestion Control Algorithm with Selective Switching features.

[5] coined the term delay-based congestion avoidance which is used as part of ACS, while admitting that his proposed algorithm was not sufficient for a practical network, his research work provided the foundation for future research on DCA including ACS. Accordingly the work described in [15] and [16] which showed that TCP/Vegas can improve TCP throughput over the Internet by avoiding packet loss, comparatively these studies were based on Internet paths, or network infrastructure that existed in the early 1990s, which generally involved at least one-T-one speed link and consequently allows any given flow to consume a significant fraction of available bandwidth. These studies were on packet loss events and also did not isolate the impact of applying the congestion avoidance algorithm.

As part of VoIPv6 packets flow performance improvement and according to [20] most VoIP equipment's up to the mid of 2002 has dynamic jitter buffers. These jitter buffers compare 
the receive time with the sent time stamp in the RTP header to calculate the jitter and automatically adjust the jitter buffer size. The installer has little control of the jitter buffer size and can only send the minimum and maximum size of data provided that the maximum jitter buffer size should be at least two times the voice sample collection time, butting in mind that the Jitter buffer size directly affects the latency in V0IPv6 experienced by the user. A jitter buffer cannot solve the problem of excessive packet jitter, and the Solutions for excessive packet jitter must be made in the network to reduce congestion and queuing time for voice packets, in this context the scope of this research work is limited to enhancement in the protocol structure purposely to improve VoIPv6 as a real-time application.

\section{B. TCP-over-UDP and TCP Switching (between Packet and Circuit Switching) Compared to This Research Work Proposed ACS}

[17] presented an interesting idea for VoIPv6 voice streaming improvement, which was TCP-over-UDP (ToU), a conditional instance of TCP on top of UDP, this idea is based on bringing TCP traffic to flow over UDP conditionally, instead of which this research work found it more practical to propose the switching idea (ACSP) between TCP and RTP conditionally. According to [18], there has been much discussion about the best way to combine the benefits of new optical circuit switching technology with the established packet switched Internet in order to explore how electronic and/or optical circuit switching might be introduced in an evolutionary manner.

[8] from the Institute of Information Science Beijing Jiaotong University of China, in their research work with the title (Congestion Control Algorithms for a new TCP/UDP router based on 2-D stability conditions), developed a congestion control algorithm for new TCP/UDP router based on 2-D stability conditions, they utilize the Dual Router idea to use both TCP and UDP, but not to switch between them conditionally as in this research work proposed ACS, and to use this to develop congestion control algorithms for the real time traffic based on switching focusing mainly on congestion avoidance as a step towards performance improvement, as in this research work an Automatic Conditional Switching (ACS) was developed to improve VoIPv6 performance. Research work by [8] can be viewed as an attempt to improve TCP performance which was used as part of ACS utilizing both RTP and Delay based TCP in order to provide smart switching capability to the VoIPv6 traffic.

According to [19], there was research effort concern in the year 2006 which had been raised about whether the increase of UDP traffic due to Skype type of applications will throttle down regular TCP users. This concern came from the fact that UDP connections are unresponsive to congestion, and accordingly UDP senders do not reduce their sending rates, which considered an incentive to investigate the fairness issue between VoIP flows and TCP flows under different network environment, considering both transport layer congestion control mechanism built-in TCP protocol stack and the selfadaptive-ness of VoIP users.

\section{CONCLUSION AND FUTURE WORKS}

ACS is a conditional switching between TCP Reno, and RTP based on the 150 milliseconds delay hit-limit standardized by the International Telecommunication Union (ITU) as in the G.114 standard recommendation (G.114 International Telecommunication Union, 1994). Without mentioning TCP due its associated delay problems, [9] stated that, currently (in the year 2014), most multimedia applications utilize a combination of real-time transport protocol (RTP) and User Datagram Protocol (UDP). In contrary this research work's novelty is a proposal to use flavored TCP Reno in one hand and Progressive RTP in the other hand, and applying Automatic Conditional Switching (ACS) between them, this ACS was tested, verified, and validated.

Developing mathematical equation and represent it with mathematical model using Laplace transform to describe the conditional switching in ACS is of concern as future work as it can be a platform for further improvements towards ACS practical implementation, and in this context a suggestion can be made to use Laplace transform to represent the switching mathematically.

Research work in [21] can be considered as base idea for future work to improve ACS, which is mainly to implement ACS in the real time, this is for a reason that they provided mathematical parallels between packet switching and information transmission. In order to conduct the tests in real time the steps presented in Fig. 1 must be followed carefully, and the following are needed:

- Analog to digital converter to produce the digitized voice in real time.

- Traffic shaper to shape the VoIPv6 traffic in IPv6.

- Perform the switching in real-time using ACS.

\section{REFERENCES}

[1] G.114, One Way Transmission, International Telecommunication Union (ITU), 1994.

[2] J. Martin, A. Nilsson, and I. Rhee, "Delay Based Congestion Avoidance for TCP", ACM Tansactions on Networking, vol. 11(3), June 2003.

[3] Keixn Ma, Ravi R, Mazumdar, and Jun Luo, "On the performance of Primal Dual Schemes for Congestion Controls in Networks with Dynamic Flow", Proceedings of 27th IEEE International Conference on Computer Communications, pp. 326-330, April, 2008.

[4] C. Hansen, "Network Performance Measurement Framework for Realtime Multiplayer Mobile Games", IEEE Network and System Support for Games, December, 2013.

[5] R. Jain, "A Delay-based Approach for Congestion Avoidance in Interconnected Heterogeneous Computer Networks", Computer. Communication Revue, vol.19(5), pp. 56-71, October, 1989.

[6] ManasPratimSarma, "Performance Measurement of TCP and UDP Using different Queuing Algorithm in High Speed Local Area Networks", International Journal of Future Computer and Communication, vol. 2(6), December, 2013.

[7] Microsoft Extensions, Real-time Transport Protocol (RTP, RTCP), Technical Documentation. Microsoft published Open Specifications documentation, May 15, 2014.

[8] Yang Xiao, Lei Wang, Jun Niu, Seok Woo, and Kiseon Kim, "Congestion Control Algorithms for a New TCP/UDP Router Based on 2-D Stability Conditions", IEEE Press Piscataway, Proceedings of the 5th International Conference on Wireless Communications, Networking and Mobile Computing, pp. 4056-4060, 2009. 
[9] W. Lei, W. Zhang, "Multipath Real-Time Transport Protocol Based on Application-Level Relay (MPRTP-AR)", Network Working Group of the Internet Engineering Task Forth (IETF), July 25, 2014.

[10] Asaad Abdallah Abusin, M D Jahangir Alam and Junaidi Abdullah. "Testing and analysis of VoIPv6, (Voice over Internet Protocol V6) performance using FreeBSD", International Journal of Computing, Networking, and System Sciences, May, 2012.

[11] Habibullah Jamal, and Kiran Sultan, "Performance Analysis of TCP Congestion Control Algorithms", International Journal of Computers and Communications, vol. 2(1). (2008),

[12] K. Egevang, RFC 1631, International Telecommunication Union (ITU). May, 1994.

[13] T. Hain, Microsoft, RFC 2993, Telecommunication Union (ITU). November, 2000.

[14] Visual Box www.virtualbox.org, https://www.virtualbox.org/ticket/5918, reported by (Clockwork). $6 / 11 / 2010$.

[15] L. S. Brakmo, S. W. O’Malley, and Peterson, "TCP Vegas: New Techniques for Congestion Detection and Avoidance", Proceedings of ACM SIGCOMM, pp. 24-35, August, 1994.

[16] J. Ahn, P. Danzig, Z. Liu, and L. Yan, "Evaluation of TCP Vegas: Emulation and Experiment", ACM SIGCOMM, pp. 185-195, 1995.

[17] S. Baset, and H. Schulzrinne, "TCP-over-UDP", Transport Area Working Group of the IETF, June, 2009.

[18] Pablo Molinero Fernández, and Nick McKeown, "TCP Switching: Exposing Circuits to IP", Stanford University (PowerPoint Presentation). 2002.

[19] Tian Bua, Yong Liub and Don Towsleyc, "On the TCP-Friendliness of VoIP Traffic", IEEE INFOCOM, 2006.
[20] Gartner Consulting, "Convergence Challenges for Enterprise Networks", Gartner Consulting White Papers, May, 2002.

[21] Tony Lee T, "The Mathematical Parallels between Packet Switching and Information Transmission", IEEE Transactions on Information Technology, October, 2006.

[22] Mark Allman, "Internet Measurement", Internet Measurement Research Group IMRG, 6 August, 2012.

[23] Luo Junhai, Chengdu Fan Mingyu, and Ye Danksia, "Research on Topology Discovery for IPv6 Networks", International Conference on Software Engineering, Artificial Intelligence, Networking, and Parallel Distributed Computing. vol. 3, page 5, 1 August, 2007.

[24] International IPv6 Conference NAV6 Malaysia, Proceedings of NAV6, Jointly organized by the University Since Malaysia USM in collaboration with university of Malaya (UM) and the International University Malaysia-Wales (IUMW), 2013.

[25] Geoff Huston, "World IPv6 Day: A year in the Life", AMPST of Australia, 10 Jun 2013

[26] http://www.technowledge.org, "A short detailed report that compares the real world performance of the most popular VOIP mobile phone", May 17, 2013.

[27] Internet drafts, TCP over UDP, IETF, June 2013.

[28] Muhand A. Mutaab, Nahla Abdul Jalil, and Jameelah H.Suad, "A Network Chatting and Sending Data", International Journal of Scientific \& Engineering Research, December, 2014.

[29] Mandakini Yayade and Sanjeev Sharma, "Review of different TCP Variants in Ad-hoc Networks", International Journal of Engineering Science and Technology (IJEST), vol. 3(3), March 2011.

[30] B. Arunakumari and P. Chennareddy, "TCP Reno, Sack and Vegas Performance Analysis", International Journal on Cybernetics \& Informatics vol. 4(2), April 2015. 